Canadian Journal of Physics

Canadian

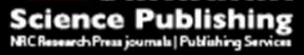

Revue canadienne de physique

\title{
AN INVESTIGATION OF SOME QUANTUM CORRELATIONS OF DIRAC FIELDS IN NON INERTIAL FRAMES
}

\begin{tabular}{|r|l|}
\hline Journal: & Canadian Journal of Physics \\
\hline Manuscript ID & cjp-2017-0489.R2 \\
\hline Manuscript Type: & Article \\
\hline Date Submitted by the Author: & 19-Feb-2018 \\
\hline Complete List of Authors: & Arjmandi, Mohammad Bagher; University of Tabriz \\
\hline Keyword: & $\begin{array}{l}\text { quantum, correlations, curve spacetime, Rindler spacetime, non inertial } \\
\text { frame }\end{array}$ \\
\hline $\begin{array}{r}\text { Is the invited manuscript for } \\
\text { consideration in a Special } \\
\text { Issue? : }\end{array}$ & N/A \\
\hline
\end{tabular}




\title{
AN INVESTIGATION OF SOME QUANTUM CORRELATIONS OF DIRAC FIELDS IN NONINERTIAL FRAMES
}

\author{
Mohammad Bagher Arjmandi \\ Faculty of Physics, Theoretical and Astrophysics Department, University of Tabriz \\ Tabriz, Iran \\ arjmandi94@gmail.com
}

\begin{abstract}
In this paper, some concepts of quantum correlations such as one-way quantum deficit, purity and entanglement of formation are investigated between the modes of a Dirac fields in noninertial frames. We consider two bipartite divisions of a tripartite system constructed by initial Werner states which is shared between two relatively accelerated observers. The degradation of these quantum correlations is shown when the acceleration parameter increases. However a non-zero amount of correlations survives even at infinite acceleration limit. As well as we study the inevitable influence of mixedness factor of initial state on the quantum correlations behaviour. Finally, we review our calculation beyond single-mode approximation and point some differences and similarities between two regims.
\end{abstract}

Keywords: Quantum correlations; Noninertial frames; Rindler spacetime.

\section{Introduction}

Quantum correlations as the applicable resources of information play a significant role in many aspects of quantum information and computation processing such as cryptography, teleportation and superdense coding $[1,2,3,4,5]$. Different kinds of quantum correlations have been studied in various areas $[6,7,8,9]$. Entanglement, the most prominent correlation, has received a considerable attention and several methods have been made to measure it, specially in case of two-qubits $[10,11]$. Moreover, quantum discord, the second remarkable quantum concept, possesses a substantial 
value which is defined based on difference between classical and nonclassical correlations $[12,13,14]$. Also the other quantum correlations like deficit, dissonance, consonance and etc. have been constructed and used under different conditions $[15,16]$. However all of mentioned works are implemented in inertial frames, so a question will be made about behaviour of quantum correlations when a noninertial observer wants to study them for quantum systems. Hence the theory of relativistic quantum information has been under widespread attention among researchers. This concentration of studies is not unreasonable because in many experimental investigations some particles like photons are used which have relativistic behaviour.

In this regard, Fuentes-Schuller et al. considered a maximal entangled state shared between two modes of a noninteracting massless scalar field when one of the observers describing the state is uniformly accelerated and showed degradation of entanglement due to relative acceleration of observers [17]. As well as Alsing et al. achieved the same results about decrease of entanglement between two modes of a free Dirac field as seen by two relatively accelerated parties [18]. In parallel manner some authors perused tripartite and multipartite entanglement in noninertial frames using $\pi$-tangle $[19,20]$. Apart from entanglement, Wang et al. studied classical correlation and quantum discord sharing of Dirac fields in noninertial frames [21]. Also A. Datta investigated quantum discord between two free modes of scalar field shared between two relative accelerated observers and confirms existence of a non zero amount of purely quantum correlations even in limit of infinite acceleration [22]. In a parallel way in Ref. [23] quantum discord and entanglement are compared by geometric measures for Dirac fields and a decreasing trend for each correlations is noticed but no completely disappearance even in infinite acceleration. In addition, some partial conservative relations for entanglement, discord and mutual information are presented under specific conditions. Similarly, quantum coherence, the main distinguishing feature of quantum mechanic from classical protocols, has been considered by Chen et al. [24] for scalar (bosonic) and Dirac (fermionic) fields. The authors used relative entropy of coherence and noticed quantum coherence degradation with increase of acceleration in both fields. Though for scalar field quantum coherence vanished with infinite acceleration, it reached to a non-zero value in Dirac field.

However, the lack of some works about other quantum correlations is still perceptible. So we aim to present an analysis about less common correlations between two modes of a Dirac field described by relatively accelerated 
observers. In this regard we begin with quantum deficit (one-way) which was first proposed by Oppenheim et al. [25] to studying thermodynamical systems and is defined as the amount of work which could be extracted from a heat bath by local operations. Then we study purity of states as a measure of decoherence indicating the degree of mixedness of quantum systems [26]. To more comparison finally we try entanglement of formation (EOF) as a measure of quantum entanglement. In most previous cases, an initial pure state is used describing twe field modes. But taking the mixedness effects of initial state into account strongly makes sense. In this regard, Xiao et al. [27] considered initial Werner state and realised that entanglement and discord positively correlate with mixedness parameter of the state. Therefore, in this paper the initial Werner state is used as a field state seen by the the observers.

The rest of the paper is organized as follows. In Section 2 we review the protocol of noninertial frames and some obligatory tools and then obtain density matrix of tripartite system. Section 3 is dedicated to introduce the quantum correlations used in this paper and next we calculate these correlations for two different bipartite states and plot them versus acceleration parameter. In Section 4 we review our calculation beyond single-mode approximation. Finally in Section 5 a summary and conclusion are prepared.

\section{Noninertial frame: The regions of Rindler spacetime}

Let consider two observers namely Alice and Rob which share an initial state of a fermionic (Dirac) field. While Alice is stationary, Rob moves respect to her with a uniform acceleration $(a)$. The well-known Unruh effect $[29,30]$ describes that when Alice sees vacuum state of the field, Rob (noninertial) will see states of a thermal bath. Here description of the problem will be divided into three modes in Rindler spacetime [28]: (i) Alice's Minkowskian mode, (ii) Rob's mode in region I of Rindler spacetime and (iii) antiRob's mode which is an unreal observer in region $I I$ and has no access to region $I$ because of acceleration horizon. Since two regions are completely disconnected then each observer must trace over inaccessible regions and therefore the information of these discarded parts could not be included in argument. Now we are ready to focus on our calculations. Using the so-called Bogoliubov coefficients one can see the vacuum and one-particle states of Rob in Minkowski spacetime are transformed to the following Rindler spacetime relations [18, 23]: 


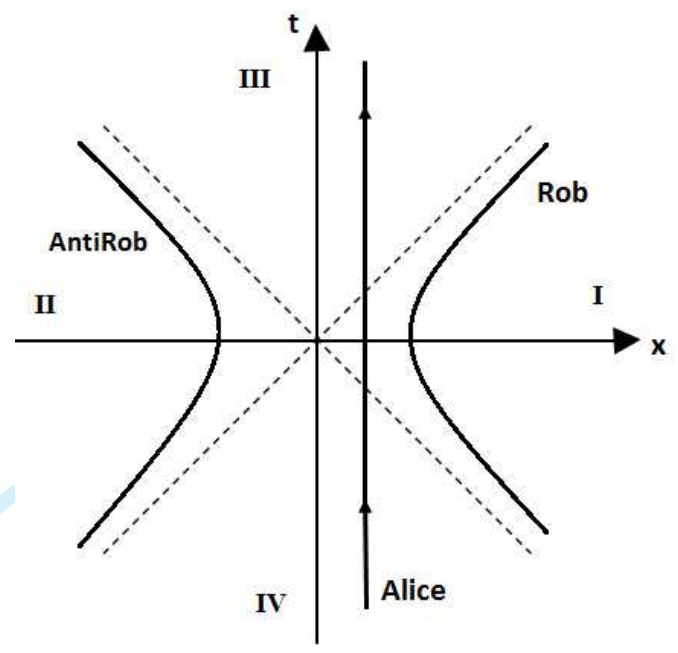

Figure 1: Rindler spacetime diagram. The event horizons (dashed) cause two regions I and II be completely disconnected.

$$
\begin{aligned}
& \left|0_{R}\right\rangle: \cos (r)\left|0_{I} 0_{I I}\right\rangle+\sin (r)\left|1_{I} 1_{I I}\right\rangle \\
& \left|1_{R}\right\rangle:\left|1_{I} 0_{I I}\right\rangle .
\end{aligned}
$$

so that $r$ denotes acceleration parameter taking values of $0 \leq r \leq \pi / 4$ for Rob acceleration $0 \leq a \leq \infty$. The subscripts $I$ and $I I$ indicate two regions of Rindler spacetime. Here as mentioned before we assume that initial field state seen by Alice and Rob is Werner state [31] given by

$$
\rho_{A B}=\frac{1-\alpha}{4} I_{2} \otimes I_{2}+\alpha\left|\Phi_{+}\right\rangle\left\langle\Phi_{+}\right|
$$

where $I_{2}$ is identity matrice of each subsystem, $\left|\Phi_{+}\right\rangle=1 / \sqrt{2}\left[\left|0_{A} 0_{B}\right\rangle+\right.$ $\left.\left|1_{A} 1_{B}\right\rangle\right]$ and $0 \leq \alpha \leq 1$ which for $\alpha=1$ the state is maximally entangled. This factor determines the purity amount of the state. Substituting relations (1) and (2) in Eq. (3) yields

$$
\begin{aligned}
\rho_{A, I, I I}= & \frac{1+\alpha}{4}\left[\cos ^{2}(r)\left|0_{A} 0_{I} 0_{I I}\right\rangle\left\langle 0_{A} 0_{I} 0_{I I}\left|+\sin ^{2}(r)\right| 0_{A} 1_{I} 1_{I I}\right\rangle\left\langle 0_{A} 1_{I} 1_{I I}\right|+\right. \\
& \cos (r) \sin (r)\left|0_{A} 0_{I} 0_{I I}\right\rangle\left\langle 0_{A} 1_{I} 1_{I I}|+\cos (r) \sin (r)| 0_{A} 1_{I} 1_{I I}\right\rangle\left\langle 0_{A} 0_{I} 0_{I I}\right|+ \\
& \left.\left|1_{A} 1_{I} 0_{I I}\right\rangle\left\langle 1_{A} 1_{I} 0_{I I}\right|\right]+\frac{\alpha}{2}\left[\cos (r)\left|0_{A} 0_{I} 0_{I I}\right\rangle\left\langle 1_{A} 1_{I} 0_{I I}\right|+\right.
\end{aligned}
$$




$$
\begin{aligned}
& \sin (r)\left|0_{A} 1_{I} 1_{I I}\right\rangle\left\langle 1_{A} 1_{I} 0_{I I}|+\cos (r)| 1_{A} 1_{I} 0_{I I}\right\rangle\left\langle 0_{A} 0_{I} 0_{I I}\right|+ \\
& \left.\sin (r)\left|1_{A} 1_{I} 0_{I I}\right\rangle\left\langle 0_{A} 1_{I} 1_{I I}\right|\right]+\frac{1-\alpha}{4}\left[\cos ^{2}(r)\left|1_{A} 0_{I} 0_{I I}\right\rangle\left\langle 1_{A} 0_{I} 0_{I I}\right|+\right. \\
& \sin ^{2}(r)\left|1_{A} 1_{I} 1_{I I}\right\rangle\left\langle 1_{A} 1_{I} 1_{I I}|+\cos (r) \sin (r)| 1_{A} 0_{I} 0_{I I}\right\rangle\left\langle 1_{A} 1_{I} 1_{I I}\right|+ \\
& \left.\cos (r) \sin (r)\left|1_{A} 1_{I} 1_{I I}\right\rangle\left\langle 1_{A} 0_{I} 0_{I I}|+| 0_{A} 1_{I} 0_{I I}\right\rangle\left\langle 0_{A} 1_{I} 0_{I I}\right|\right]
\end{aligned}
$$

that is a tripartite state consisting of Alice (A), Rob (I) and AntiRob (II) subsystems. Now for each subsystem by tracing over inaccessible modes three different bipartitions Alice-Rob, Alice-antiRob and Rob-antiRob can be obtained. Because of event horizon and disconnection of regions $I$ and $I I$ the last bipartition is meaningless. So let us to ignore it and pay attention to other two bipartite states which are

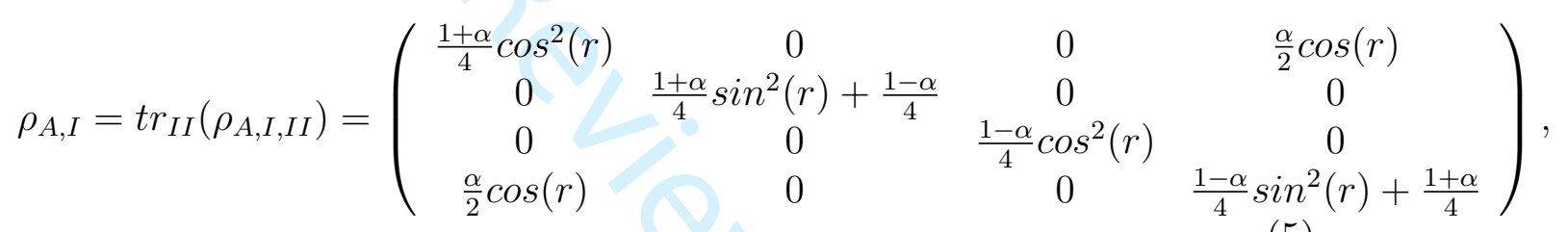

and

$\rho_{A, I I}=\operatorname{tr}_{I}\left(\rho_{A, I, I I}\right)=\left(\begin{array}{cccc}\frac{1+\alpha}{4} \cos ^{2}(r)+\frac{1-\alpha}{4} & 0 & 0 & 0 \\ 0 & \frac{1+\alpha}{4} \sin ^{2}(r) & \frac{\alpha}{2} \sin (r) & 0 \\ 0 & \frac{\alpha}{2} \sin (r) & \frac{1-\alpha}{4} \cos ^{2}(r)+\frac{1+\alpha}{4} & 0 \\ 0 & 0 & 0 & \frac{1-\alpha}{4} \sin ^{2}(r)\end{array}\right)$.

Having these states we are able to calculate different quntum correlations and compare them that is the subject of the next section.

\section{Quantum correlation}

In this section we review three quantum correlations (deficit, eof and purity) and then investigate these functions versus acceleration parameter $(r)$ for different values of $\alpha$. 


\subsection{Quantum deficit}

Quantum deficit which at first was introduced to studying thermodynamical systems is defined as the amount of extractable work from quantum systems coupled to a heat bath by local operations and classical communication (LOCC). Depending on classical communication between the systems three types of quantum deficit namely zero, one and two-way deficit could be generated. For bipartite state $\rho_{A B}$ consisting of two subsystems $A$ and $B$, the one-way quantum deficit considered in this paper is defined by $[7,15]$

$$
d\left(\rho_{A B}\right)=\min _{\left(\Pi_{j}^{A}\right)} s\left(\rho_{A B}^{\prime}\right)-s\left(\rho_{A B}\right),
$$

where $\rho_{A B}^{\prime}=\sum_{j}\left(\Pi_{j}^{A} \otimes I_{B}\right) \rho_{A B}\left(\Pi_{j}^{A} \otimes I_{B}\right)$ is the state after performing measurement on subsystem $A$, and $s(\rho)=-\operatorname{Tr} \rho \log _{2} \rho$ is the von Neumann entropy of state $\rho$. The minimum is taken over all possible projective measurements : $\Pi_{j}^{A}=\left|j^{\prime A}\right\rangle\left\langle j^{\prime A}\right|, j=\{0,1\}$ where

$$
\begin{aligned}
\left|0^{\prime}\right\rangle & =\cos \left(\frac{\theta}{2}\right)|0\rangle-e^{-i \phi} \sin \left(\frac{\theta}{2}\right)|1\rangle, \\
\left|1^{\prime}\right\rangle & =e^{i \phi} \sin \left(\frac{\theta}{2}\right)|0\rangle+\cos \left(\frac{\theta}{2}\right)|1\rangle,
\end{aligned}
$$

and the measurement operators satisfy $\Pi_{j}^{A} \geq 0$ and $\sum_{j} \Pi_{j}^{A}=I$. The mentioned minimization depends on $\theta$ and $\phi$ which are the angles of Bloch sphere and take the values $\theta \in[0, \pi]$ and $\phi \in[0,2 \pi)$. Consider

$$
\delta(\theta, \phi)=s\left(\sum_{j}\left(\Pi_{j}^{A} \otimes I_{B}\right) \rho_{A B}\left(\Pi_{j}^{A} \otimes I_{B}\right)\right)
$$

Here, to obtain the optimal measurements, we only need to minimize $\delta(\theta, 0)$. The first partial derivative of $\delta(\theta, 0)$ with respect to $\theta\left(\frac{\partial \delta(\theta, 0)}{\partial \theta}=\right.$ $0)$ gives the extreme points and the second derivative $\left(\frac{\partial^{2} \delta(\theta, 0)}{\partial \theta^{2}}\right)$ determines whether these points are minimum or maximum. Ultimately, the one-way deficit for two states (5) and (6) is obtained as

$$
\begin{aligned}
d\left(\rho_{A I}\right)= & \frac{1}{16 \log (2)}\left[-4(-1+\alpha) \cos ^{2}(r) \log \left(-\frac{1}{4}(-1+\alpha) \cos ^{2}(r)\right)+\right. \\
& 2\left(-4+a_{+}\right) \log \left(\frac{1}{16}\left(4-a_{+}\right)\right)-2\left(4+a_{+}\right) \log \left(\frac{1}{16}\left(4+a_{+}\right)\right)+
\end{aligned}
$$




$$
\begin{aligned}
& \left(c_{+}-b_{+}\right) \log \left(\frac{1}{16}\left(c_{+}-b_{+}\right)\right)+\left(c_{+}+b_{+}\right) \log \left(\frac{1}{16}\left(c_{+}+b_{+}\right)\right) \\
& \left.-2(-3+\alpha+(1+\alpha) \cos (2 r)) \log \left(\frac{1}{4}\left(1-\alpha+(1+\alpha) \sin ^{2}(r)\right)\right)\right]
\end{aligned}
$$

$$
\begin{aligned}
d\left(\rho_{A I I}\right)= & \frac{1}{16 \log (2)}\left[2(3-\alpha+(1+\alpha) \cos (2 r)) \log \left(\frac{1}{8}(3-\alpha+(1+\alpha) \cos (2 r))\right)\right. \\
& +\left(c_{-}-b_{-}\right) \log \left(\frac{1}{16}\left(c_{-}-b_{-}\right)\right)+\left(c_{-}+b_{-}\right) \log \left(\frac{1}{16}\left(c_{-}+b_{-}\right)\right)+ \\
& 2\left(-4+a_{-}\right) \log \left(\frac{1}{16}\left(4-a_{-}\right)\right)-2\left(4+a_{-}\right) \log \left(\frac{1}{16}\left(4+a_{-}\right)\right)- \\
& \left.4(-1+\alpha) \sin ^{2}(r) \log \left(-\frac{1}{4}(-1+\alpha) \sin ^{2}(r)\right)\right]
\end{aligned}
$$

where

$$
\begin{gathered}
a_{ \pm}=(2)^{1 / 2}\left(3+4 \alpha^{2} \pm 4\left(-1+\alpha^{2}\right) \cos (2 r)+\cos (4 r)\right)^{1 / 2} \\
b_{ \pm}=(2)^{1 / 2}\left(3+16 \alpha^{2}+4\left(\mp 1 \pm 4 \alpha^{2}\right) \cos (2 r)+\cos (4 r)\right)^{1 / 2} \\
c_{ \pm}=2(2+\alpha \pm \alpha \cos (2 r)) .
\end{gathered}
$$

In figure 2 we plot the one-way deficit of Alice-Rob state for three different values of $\alpha$. All three curves confirm the degradation of deficit when acceleration increases. However, as we expect for Dirac fields, some degree of this correlation survives even at the maximum value of $r$ i.e. $a \rightarrow \infty$. This means that we can rely on quantum deficit as a suitable candidate of information resource even in infinite acceleration limit.

Figure 3 shows the influence of $r$ and $\alpha$ on deficit simultaneously. As we already said, $d\left(\rho_{A I}\right)$ has a decreasing trend when $r$ increase. In contrast, it gradually goes up with growth of $\alpha$ meaning that the more pure the initial state is, the greater deficit will be. Meanwhile, if $\alpha=0$ (completely mixed state), then $d\left(\rho_{A I}\right)=0$ for any $r$.

In the two next figures, we focus on Alice-antiRob state. As seen from figure 4 at $r=0, \rho_{A I I}$ has no any amount of quantum deficit, no matter how big $\alpha$ is. Then, it begins to revive with a rate based on $\alpha$ so that $d\left(\rho_{A I I}\right)$ sharply grows with increase of acceleration for bigger amounts of $\alpha$. Figure 5 
properly compares the opposite behaviour of $d\left(\rho_{A I}\right)$ and $d\left(\rho_{A I I}\right)$ versus $r$. It seems that the quantum correlation is transfered through the event horizon from reigin $I$ to $I I$.

\subsection{Purity}

Purity as a measure of coherence indicating the degree of mixedness of states is defined by $[32,33]$

$$
p=\operatorname{Tr}\left[\rho^{2}\right]
$$

for any arbitrary state $\rho$. It equals to one for pure states wheras leads to its minimum for maximally mixed state. In the other word for $\rho=\frac{I}{N}$ purity is $\frac{1}{N}$ which $N$ is the dimension of the system. Using (5), (6) and (12) we have:

$$
\begin{aligned}
& p\left(\rho_{A I}\right)=\frac{1}{32}\left[4\left(3 \alpha^{2}-1\right) \cos (2 r)+\left(1+\alpha^{2}\right)(\cos (4 r)+11)\right] \\
& p\left(\rho_{A I I}\right)=\frac{1}{32}\left[4\left(1-3 \alpha^{2}\right) \cos (2 r)+\left(1+\alpha^{2}\right)(\cos (4 r)+11)\right] .
\end{aligned}
$$

Figure 6 shows the purity of Alice-Rob bipartition state for different $\alpha$. Here, for $\alpha=0.8, p\left(\rho_{A I}\right)$ shows more decay meaning that $\rho_{A I}$ leads to lose coherence and become more mixed. However, for $\alpha=0.3,0.5$ there is a slight degradation and next the curves revive at the top limit of acceleration. Hence, some defree of $p\left(\rho_{A I}\right)$ resist in maximal acceleration. It is worthwhile to see how $r$ and $\alpha$ contribute in behaviour of $p\left(\rho_{A I}\right)$ (figure 7 ). Again, we can obviously see the importance of $\alpha$ which correlates positively with degree of coherence. But as we mentioned before, in Ref. [24] quantum coherence has been studied for scalar and Dirac fields by using the relative entropy of coherence. There for Dirac field, quantum coherence had a falling trend with increase of acceleration. But interestingly, it fluctuated with $\alpha$ so that reached to it's mazimum amount and then fell to zero.

In figure 8 we plot the purity of Alice-antiRob state versus $r$ for three different $\alpha$. The start point of all three curves is 0.5 which means that at $r=0$ the initial purity of $\rho_{A I I}$ is $\frac{1}{2}$, regardless of $\alpha$. But purity degradation rate is completely $\alpha$ dependant. Comparing $p\left(\rho_{A I}\right)$ and $p\left(\rho_{A I I}\right)$ in figures 6 and $8, \alpha$ plays a significant but different role in each diagram. In figure 6 , the 
curves with bigger $\alpha$ undergo more decay and those with smaller $\alpha$ first have a slight fall and then begin to increase in high acceleration. On the other hand, $p\left(\rho_{A I I}\right)$ diminishes more for slighter $\alpha$. Figure 9 represents a good comparison between $p\left(\rho_{A I}\right)$ and $p\left(\rho_{A I I}\right)$ for $\alpha=0.5$. To some extent, both functions have a similar trend and the final values are almost the same (just a small difference).

\subsection{Entanglement}

Now we study entanglement of the states by entanglement of formation (eof) as a measure applied on mixed entangle states that for composite state $\rho$ is defined by the minimum average entanglement of an ensemble of pure states indicating $\rho$. Acording to Wootters definition [10] the eof reads

$$
e o f=-\frac{1+\sqrt{1-c^{2}}}{2} \log _{2}\left(\frac{1+\sqrt{1-c^{2}}}{2}\right)-\frac{1-\sqrt{1-c^{2}}}{2} \log _{2}\left(\frac{1-\sqrt{1-c^{2}}}{2}\right)
$$

where $c$ denotes the concurrence

$$
c(\rho)=\max \left\{0, \lambda_{1}-\lambda_{2}-\lambda_{3}-\lambda_{4}\right\},
$$

so that $\lambda_{i}$ (with condition: $\lambda_{i}>\lambda_{i+1}$ ) are the square roots of the eigenvalues of $\rho \widetilde{\rho}$ which $\widetilde{\rho}=\left(\sigma_{y} \otimes \sigma_{y}\right) \rho\left(\sigma_{y} \otimes \sigma_{y}\right)$ and $\sigma_{y}$ is $Y$ component of Pauli operators. For two states (5) and (6) we have:

$$
\begin{gathered}
c\left(\rho_{A I}\right)=\frac{1}{32}\left[-(-1+\alpha) \cos ^{2}(r)(-3+\alpha+(1+\alpha) \cos (2 r))+m_{-}-m_{+}\right], \\
c\left(\rho_{A I I}\right)=\frac{1}{8}\left(m_{-}^{\prime}+m_{+}^{\prime}\right),
\end{gathered}
$$

so that

$$
\begin{aligned}
m_{ \pm}= & 4(\cos (r)((5+\alpha(8+19 \alpha)) \cos (r)) \pm \\
& 8 \sqrt{2} \sqrt{\alpha^{2}(1+\alpha) \cos ^{2}(r)(3+\alpha(-1+\alpha) \cos (2 r))} \\
& \left.+\left(-1+\alpha^{2}\right) \cos (3 r)\right)^{1 / 2}
\end{aligned}
$$




$$
\begin{aligned}
m_{ \pm}^{\prime}= & -(\sin (r)((5+\alpha(8+19 \alpha))) \sin (r) \pm \\
& 8 \sqrt{2} \sqrt{\alpha^{2}\left(3+4 \alpha+\alpha^{2}-\left(-1+\alpha^{2}\right) \cos (2 r)\right) \sin ^{2}(r)} \\
& \left.-\left(-1+\alpha^{2}\right) \sin (3 r)\right)^{1 / 2}
\end{aligned}
$$

We repeat our considerations for entanglement of formation in figures 10-13. As for $\alpha<\frac{1}{3}$ concurrence and consequntly eof is equal to zero, so we plot it for $\alpha=0.5,0.8$. Once again, we notice the degradation of eof with increase of acceleration parameter. However, a non-zero amount of entanglement remains at infinite acceleration. This results confirm previous works in this area $[22,23]$. 3D plot of eof is prepared in figure 11. Like two prior investigated cases, a rising trend can be seen for eof versus $\alpha$. As it is expected eof $\left(\rho_{A I I}\right)$ increase with growth of $r$. Also here the rate of increase is related to $\alpha$ and entanglement grows more quickly for $\alpha=0.8$ than $\alpha=0.5$. Therefore, as we can see in Ref. [27] the parameter $\alpha$ which determines the purity of initial Werner state plays an important role in behaviour of the correlations. Finally, we compare entanglement for Alice-Rob and Alice-anti-Rob states in figure 13. Although we do not see an exact conservative law between two states, but clearly entanglement is transfered from reigon $I$ to $I I$.

Here, it is worthwhile to shortly review Ref. [34] and make a comparison with our work. There the authors calculated quantum discord, logarithmic nagativity and entanglement of formation for three different bipartition Alice-Rob, Alice-antiRob and RobantiRob under single-mode approximation. Like us also in that work the initial Werner state is considered as the state of two Dirac modes shared by Alice and Rob. Needless to say, for entanglement of formation the results are exactly the same. As everyone knows, in inertial frames the initial Werener state is entangled if $\alpha>\frac{1}{3}$. Howevere, as can be seen from both papers, in infinite acceleration limit the Alice-Rob state is entangled for $\alpha>\frac{3}{7}$. But for quantum discord (Ref. [34]) and quantum deficit (in this paper), we see no critical range for $\alpha$ and both increase monotonically when $\alpha$ rises. 


\section{Beyond single-mode approximation}

The single-mode approximation aims to connect a single Minkowski frequency mode (seen by inertial observer) to a single Rindler frequency mode (seen by non-inertial observer). In the other word, each observer only detects a single frequency mode. However, This assumption is valid for specific kind of states with Minkowski wave packets and not for general states [35]. In this regard, it is worthwhile to go beyond the single-mode approximation and modify our calculations.

The Unruh vacuum state in mode $\Omega$ is defined as a product state of right and left Unruh vacua i.e. $\left|0_{\Omega}\right\rangle_{U}=\left|0_{\Omega}\right\rangle_{R} \otimes\left|0_{\Omega}\right\rangle_{L}$. When Rob accelerates, each of this Unruh states can be obtained in terms of Rindler reigons $I$ and $I I$ as follows [35]

$$
\begin{aligned}
& \left|0_{\Omega}\right\rangle_{R}=\cos (r)\left|0_{\Omega}\right\rangle_{I}^{+}\left|0_{\Omega}\right\rangle_{I I}^{-}+\sin (r)\left|1_{\Omega}\right\rangle_{I}^{+}\left|1_{\Omega}\right\rangle_{I I}^{-}, \\
& \left|0_{\Omega}\right\rangle_{L}=\cos (r)\left|0_{\Omega}\right\rangle_{I}^{-}\left|0_{\Omega}\right\rangle_{I I}^{+}+\sin (r)\left|1_{\Omega}\right\rangle_{I}^{-}\left|1_{\Omega}\right\rangle_{I I}^{+},
\end{aligned}
$$

where the superscripts \pm show the particle and antiparticle modes. Using these relations, the Unruh vacuum state can be given by

$$
\begin{aligned}
\left|0_{\Omega}\right\rangle_{R}= & \cos ^{2}(r)|0000\rangle-\sin (r) \cos (r)|0011\rangle+ \\
& \sin (r) \cos (r)|1100\rangle-\sin ^{2}(r)|1111\rangle,
\end{aligned}
$$

where we use the compact notation $|a b c d\rangle=\left|a_{\Omega}\right\rangle_{I}^{+}\left|b_{\Omega}\right\rangle_{I I}^{-}\left|c_{\Omega}\right\rangle_{I}^{-}\left|d_{\Omega}\right\rangle_{I I}^{+}$. By applying the Unruh creation operator on the Unruh vacuum state, the particle and antiparticle states have the form

$$
\begin{aligned}
\left|1_{\Omega}\right\rangle_{U}^{+}= & q_{R}(\cos (r)|1000\rangle-\sin (r)|1011\rangle)+ \\
& q_{L}(\sin (r)|1101\rangle+\cos (r)|0001\rangle), \\
\left|1_{\Omega}\right\rangle_{U}^{-}= & q_{L}(\cos (r)|0100\rangle-\sin (r)|0111\rangle)+ \\
& q_{R}(\sin (r)|1110\rangle+\cos (r)|0010\rangle),
\end{aligned}
$$

where $q_{R}$ and $q_{L}$ are complex numbers satisfying $\left|q_{R}\right|^{2}+\left|q_{L}\right|^{2}=1$. Now, substituting relations (24), (25) and (26) in (3) we can obtain the total state 
of Alice, Rob and antiRob. Here, we assume that Rob's detector is only sensitive to one-particle state $\left(\left|1_{\Omega}\right\rangle_{U}^{+}\right)$. So the antiparticle state $\left|1_{\Omega}\right\rangle_{U}^{-}$has to be traced out. Finally, tracing over $I I(I)$ reigons, the Alice-Rob (Alice-antiRob) states can be expressed as

$$
\begin{aligned}
\rho_{A I^{+}}= & {\left[\frac{1}{4}\left(2+q_{R}^{2}(-1+\alpha)\right) \cos ^{2}(r)\right]|00\rangle\langle 00|+\frac{\alpha}{2} q_{R} \cos (r)(|00\rangle\langle 11|+| 11\rangle\langle 00|)+} \\
& {\left[\frac{1}{8}\left(2-q_{R}^{2}(-1+\alpha)+\left(-2-q_{R}^{2}(-1+\alpha)\right) \cos (2 r)\right)\right]|01\rangle\langle 01|+} \\
& {\left[\frac{1}{8} \cos ^{2}(r)\left(3+\alpha-2 q_{R}^{2}(1+\alpha)-(-1+\alpha) \cos (2 r)\right)\right]|10\rangle\langle 10|+} \\
& {\left[\frac{1}{8}\left(2+q_{R}^{2}(1+\alpha)+\left(-2+q_{R}^{2}(1+\alpha)\right) \cos (2 r)\right)\right]|11\rangle\langle 11|, } \\
\rho_{A I I^{+}}= & {\left[\frac{1}{8}\left(2-q_{R}^{2}(-1+\alpha)+\left(2+q_{R}^{2}(-1+\alpha)\right) \cos (2 r)\right)\right]|00\rangle\langle 00|+} \\
& {\left[\frac{1}{4}\left(2+q_{R}^{2}(-1+\alpha)\right) \sin ^{2}(r)\right]|01\rangle\langle 01|+\frac{\alpha}{2} q_{R} \sin (r)(|01\rangle\langle 10|+| 10\rangle\langle 01|)+} \\
& {\left[\frac{1}{8}\left(2+q_{R}^{2}(1+\alpha)-\left(-2+q_{R}^{2}(1+\alpha)\right) \cos (2 r)\right)\right]|10\rangle\langle 10|-} \\
& {\left[\frac{1}{4}\left(-2+q_{R}^{2}(1+\alpha)\right) \sin ^{2}(r)\right]|11\rangle\langle 11| . }
\end{aligned}
$$

Now, for these bipartite states we can calculate quantum deficit, purity and entanglement of formation. In figure 14, we have plotted the one-way quantum deficit for Alice-Rob and Alice-antiRob states. As can be seen from these figure, the results are fairly similar to previous ones. Here also, the quantum deficit of $\rho_{A I^{+}}$degrades with increase of acceleration with a rate depending on $\alpha$, but some degree of deficit remains at infinite acceleration. Likewise, for Alice-antiRob bipartition, there is an increasing behaviour in deficit.

In the next figure, we choose a fixed value of $\alpha$ and plot the quantum deficit versus $r$ for different values of $q_{R}$. As it is expected, deficit correlates positively with $q_{R}$, meaning that the bigger $q_{R}$ is, the greater deficit will be. For special case of $q_{R}=1$, the curve is exactly the same as what we obtained formerly.

Figure 16 shows purity of $\rho_{A I^{+}}$and $\rho_{A I I^{+}}$. Here, we see a considerable difference for both Alice-Rob Alice-antiRob, compared to previous diagrams. 
For Alice-Rob state, there are different values of initial purity (based on $\alpha$ ). Then the purity decreases with increase of acceleration parameter and the state tends to be more mixed. But there is a non-zero degree of purity even at infinite acceleration limits.

On the other hand, for Alice-antiRob state the situation is rather different. The initial purity for all three cases is the same, regardless of $\alpha$, like the case under single-mode approximation. Here again the state significantly lose its purity by increase of acceleration. Interestingly, for all three values of $\alpha$ the trend is quite similar with only a small difference at high accelerations. For mor comparison, we plot the purity of these states for a fixed $\alpha$ and different values of $q_{R}$. For $q_{R}=1$ the result is exactly the same as previous diagrams. Finally, we review the entanglement of formation in figure 18. Here the eof amount of Ali-Rob state is less than the previous case calculated with singlemode approximation. Moreover, we noticed a steeper decline in entanglement before but here, it falls moderately but there is no complete disappearance of entanglement. For Alice-antiRob state also we can see a similar bahaviour to previous results.

\section{Summary and Conclusion}

In this paper, we investigated one-way deficit, purity and entanglement of formation for two typical modes of Dirac field. We chose the well-known Werner state as the initial state so that we were able to control the degree of state mixedness $(\alpha)$. For two different bipartitions Alice-Rob in reigon $I$ and Alice-antiRob in reigon II of Rindler spacetime we calculated our quantum functions versus $\alpha$ and acceleration parameter. Eventhough we notice a decreasing trend for all three quantum features in Alice-Rob state when acceleration parameter increases, but all three features survives even at infinite acceleration, reaching to non-zero amounts. This point provides a suitable resource of information based on such quantum features at high acceleration. Meanwhile in other bipartition namely Alice-antiRob, we see a considerable increasing behaviour of the correlations and purity with acceleration. More interestingly, the mixedness parameter of initial state $(\alpha)$ has a key role so that all features of both bipartitions rise with growth of $\alpha$. Thus, we have a controllable factor in initial state to determine the changes in quantum correlations.

Finally, we repeated our calculations beyond single-mode approximation. Generally, for quantum deficit and entanglement the results were similar 
to previous results with only a small difference in rate. But the changes in purity were more considerable copared to the case under single-mode approximation.

\section{References}

[1] Jennewein, T., Weihs, G., Pan, J. W., Zeilinger, A.: Experimental Nonlocality Proof of Quantum Teleportation and Entanglement Swapping. Physical review letters. 88, 017903 (2001)

[2] Bennett, C.H., Brassard, G.: In Proceedings of IEEE International Conference on Computers, Systems and Signal Processing, Bangalore, India, p. 75. New York: IEEE (1984)

[3] Ekert, A.K.: Quantum cryptography based on Bell's theorem. Phys. Rev. Lett. 67, 661 (1991)

[4] Bennett, C.H., Brassard, G., Crpeau, C., Jozsa, R., Peres, A.,Wootters,W.K.: Teleporting an unknown quantum state via dual classical and Einstein-Podolsky-Rosen channels. Phys. Rev. Lett. 70, 1895 (1993)

[5] Oh, S., Lee, S., Lee, H.W.: Fidelity of quantum teleportation through noisy channels. Phys. Rev. A 66, 022316 (2002)

[6] Franco, R. L., Bellomo, B., Maniscalco, S., Compagno, G.: Dynamics of quantum correlations in two-qubit systems within non-Markovian environments. International Journal of Modern Physics B. 27, 1345053 (2013)

[7] Streltsov, A., Kampermann, H., BruB, D.: Behavior of quantum correlations under local noise. Physical review letters. 107,170502 (2011)

[8] Aaronson, B., Franco, R. L., Adesso, G.: Comparative investigation of the freezing phenomena for quantum correlations under nondissipative decoherence. Physical Review A. 88, 012120 (2013)

[9] Mohammadi, H.: Post Markovian Dynamics of Quantum Correlations: Entanglement versus Discord. Quantum Information Processing. 16, 39 (2017) 
[10] Wootters, W. K.: Entanglement of formation of an arbitrary state of two qubits. Physical Review Letters. 80, 2245 (1998)

[11] Horodecki, R., Horodecki, P., Horodecki, M., Horodecki, K.: Quantum entanglement. Reviews of modern physics. 81, 865 (2009)

[12] Ollivier, H., Zurek, W. H.: Quantum discord: a measure of the quantumness of correlations. Physical review letters. 88, 017901 (2001)

[13] Ali, M., Rau, A. R. P., Alber, G.: Quantum discord for two-qubit X states. Physical Review A. 81, 042105 (2010)

[14] Mahdian, M., Arjmandi, M. B.: Comparison of quantum discord and relative entropy in some bipartite quantum systems. Quantum Information Processing. 15, 1569 (2016)

[15] Modi, K., Brodutch, A., Cable, H., Paterek, T., Vedral, V.: The classical-quantum boundary for correlations: discord and related measures. Reviews of Modern Physics. 84, 1655 (2012)

[16] Pei, P., Wang, W., Li, C., Song, H. S.: Using nonlocal coherence to quantify quantum correlation. International Journal of Theoretical Physics. 51, 3350 (2012)

[17] Fuentes-Schuller, I., Mann, R. B.: Alice falls into a black hole: entanglement in noninertial frames. Physical review letters. 95, 120404 (2005)

[18] Alsing, P. M., Fuentes-Schuller, I., Mann, R. B., Tessier, T. E.: Entanglement of Dirac fields in noninertial frames. Physical Review A. 74, 032326 (2006)

[19] Hwang, M. R., Park, D., Jung, E.: Tripartite entanglement in a noninertial frame. Physical Review A. 83, 012111 (2011)

[20] Wang, J., Jing, J.: Multipartite entanglement of fermionic systems in noninertial frames. Physical Review A. 83, 022314 (2011)

[21] Wang, J., Deng, J., Jing, J.: Classical correlation and quantum discord sharing of Dirac fields in noninertial frames. Physical Review A. 81, $052120(2010)$ 
[22] Datta, A.: Quantum discord between relatively accelerated observers. Physical Review A. 80, 052304 (2009)

[23] Qiang, W. C., Zhang, H. P., Zhang, L.: Geometric global quantum discord of two-qubit X states. International Journal of Theoretical Physics. 55, $1833(2016)$

[24] Chen, X., Wu, C., Su, H. Y., Ren, C. L., Chen, J. L.: Bipartite quantum coherence in noninertial frames. arXiv preprint. arXiv: 1601.02741 (2016)

[25] Oppenheim, J., Horodecki, M., Horodecki, P., Horodecki, R.: Thermodynamical approach to quantifying quantum correlations. Phys. Rev. Lett. 89, 180402 (2002)

[26] Ziman, M., Buzek,V.:Concurrence versus purity: Influence of local channels on Bell states of two qubits. Physical Review A. 72, 052325 (2005)

[27] Xiao, X., Fang, M. F.: Mixed-state entanglement in noninertial frames. Journal of Physics A: Mathematical and Theoretical. 44, 145306 (2011)

[28] Martin-Martinez, E., Leon, J.:Quantum correlations through event horizons: Fermionic versus bosonic entanglement. Physical Review A. 81, $032320(2010)$

[29] Unruh, W. G.: Notes on black-hole evaporation. Physical Review D. 14, $870(1976)$

[30] Crispino, L. C., Higuchi, A., Matsas, G. E.: The Unruh effect and its applications. Reviews of Modern Physics. 80, 787 (2008)

[31] Werner, R. F.: Quantum states with Einstein-Podolsky-Rosen correlations admitting a hidden-variable model. Physical Review A. 40, 4277 (1989)

[32] Vathsan, R.: Introduction to quantum physics and information processing. CRC Press (2015)

[33] Nielsen, M. A., Chuang, I. L.: Quantum Computation and Quantum Information. Cambridge University Press, Cambridge (2010) 
[34] Mehri-Dehnavi, H., Mirza, B., Mohammadzadeh, H., Rahimi, R.: Pseudo-entanglement evaluated in noninertial frames. Annals of Physics. 326, 1320 (2011)

[35] Bruschi, D. E., Louko, J., Martin-Martinez, E., Dragan, A., Fuentes, I.: Unruh effect in quantum information beyond the single-mode approximation. Physical Review A. 82, 042332 (2010) 


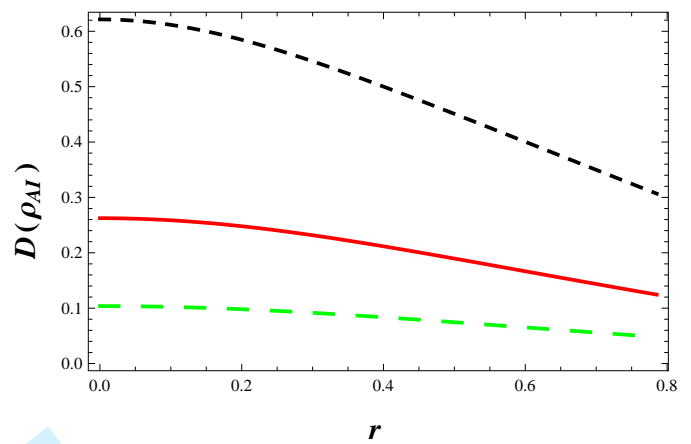

Figure 2: One-way deficit of $\rho_{A I}$ versus acceleration parameter with three values of $\alpha: 0.3$ (green large dashed), 0.5 (solid red) and 0.8 (black tiny dashed).

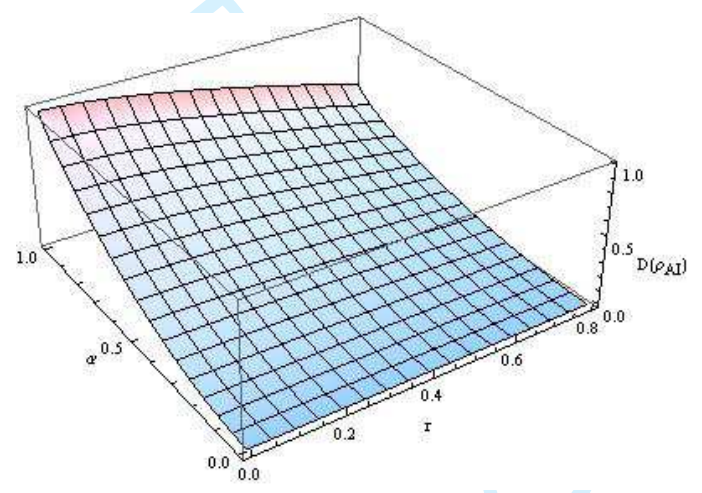

Figure 3: One-way deficit of Alice-Rob bipartition versus $r$ and $\alpha$.

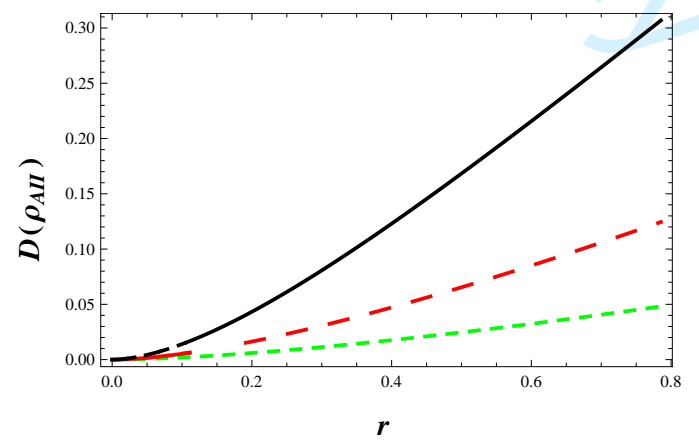

Figure 4: One-way deficit of $\rho_{A I I}$ versus acceleration parameter with three values of $\alpha$ : 0.3 (green dashed), 0.5 (red large dashed) and 0.8 (solid black). 


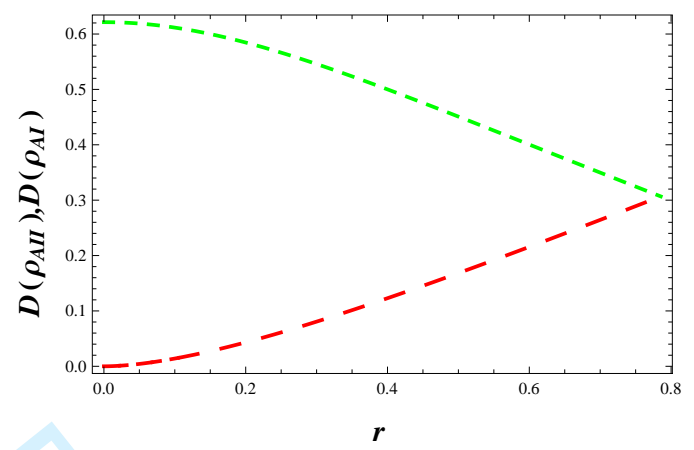

Figure 5: Comparison of one-way deficit for Alice-Rob (green dashed) and Alice-AntiRob (red large dashed) states for $\alpha=0.5$.

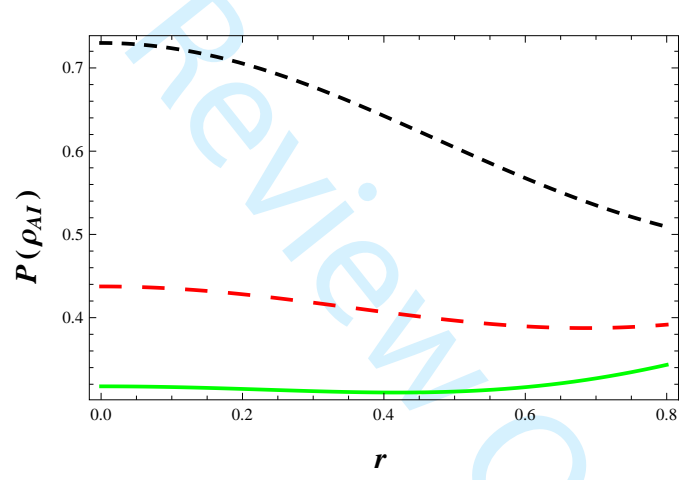

Figure 6: Purity of Alice-Rob state versus acceleration parameter. $\alpha: 0.3$ (solid green), 0.5 (red large dashed) and 0.8 (black tiny dashed).

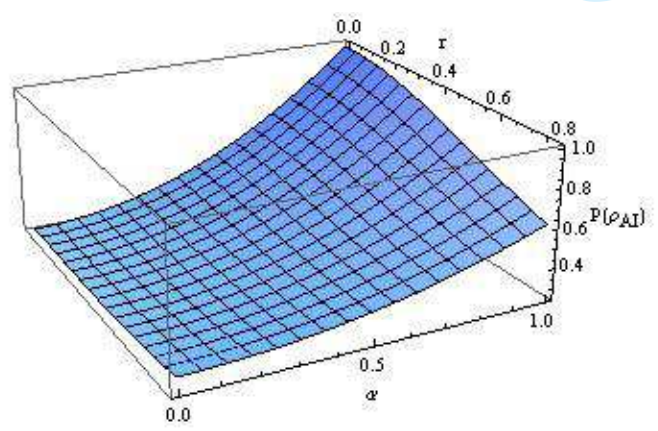

Figure 7: Purity of $\rho_{A I}$ vesus $r$ and $\alpha$. 


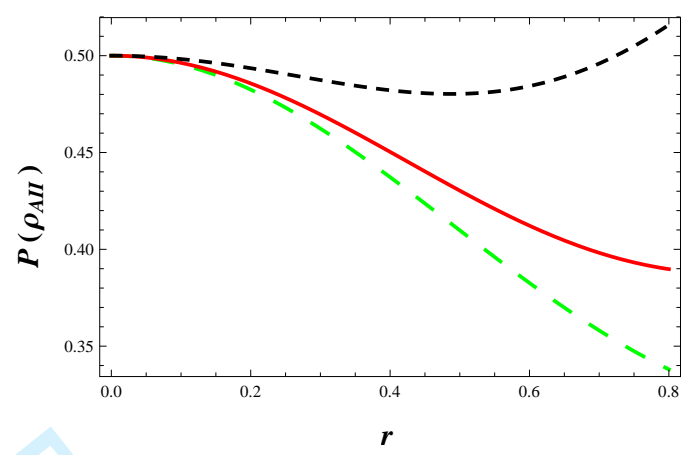

Figure 8: Purity of Alice-AntiRob state versus acceleration parameter. $\alpha: 0.3$ (green large dashed), 0.5 (solid red) and 0.8 (black tiny dashed).

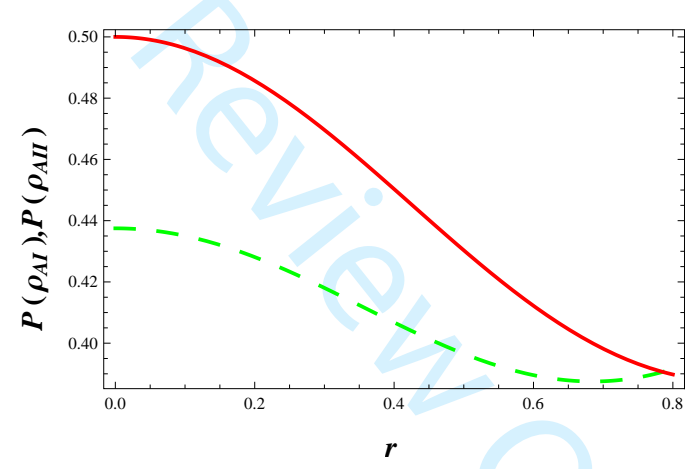

Figure 9: Purity of $\rho_{A I}$ (green dashed) and $\rho_{A I I}$ (solid red) for $\alpha=0.5$.

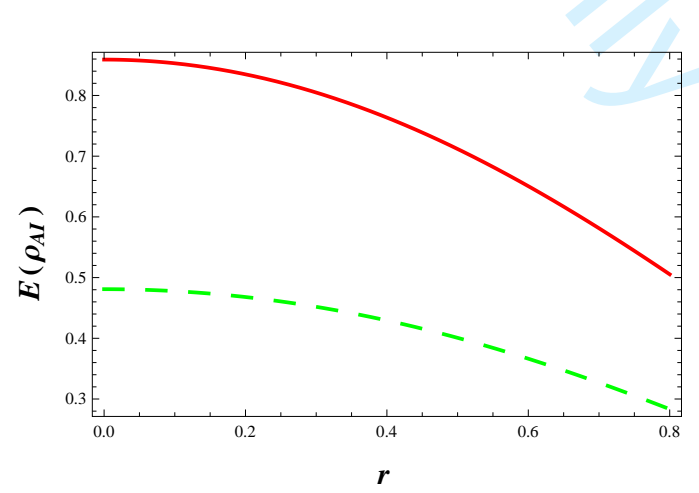

Figure 10: Entanglement of formation as a function of $r$ for Alic-Rob state. $\alpha: 0.5$ (green dashed) and 0.8 (solid red). 


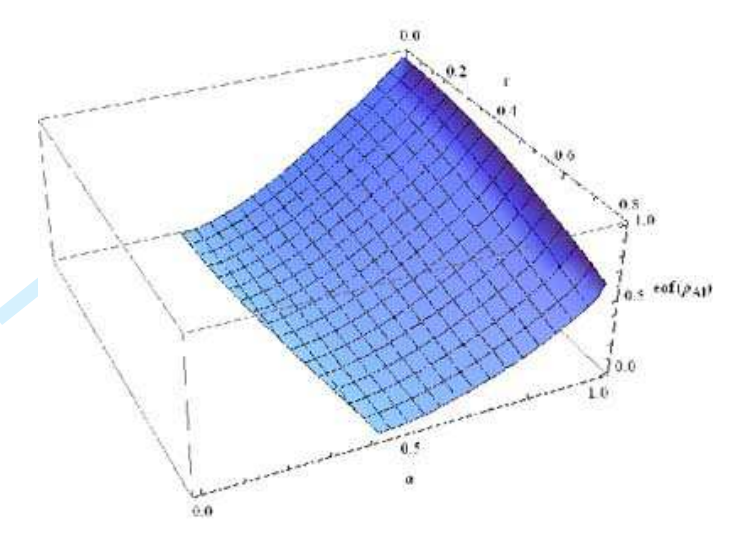

Figure 11: EOF of Alice-Rob bipartition versus $r$ and $\alpha$.

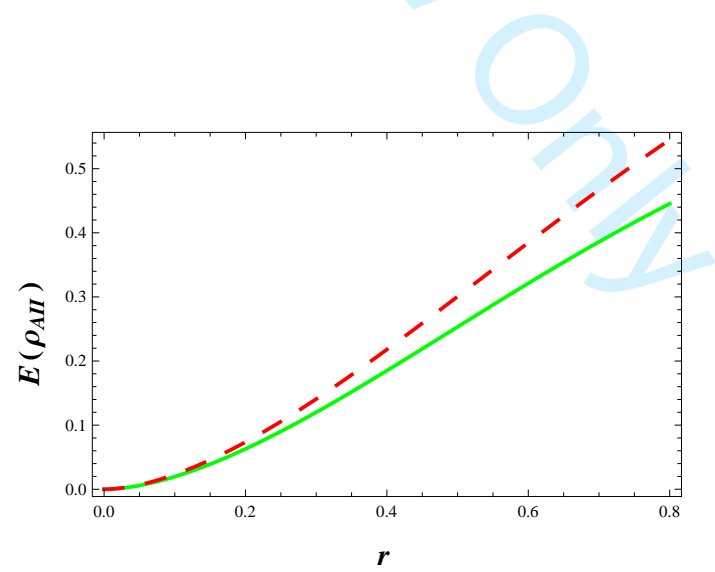

Figure 12: Entanglement of formation as a function of $r$ for Alice-AntiRob state. $\alpha: 0.5$ (green dashed) and 0.8 (solid red). 


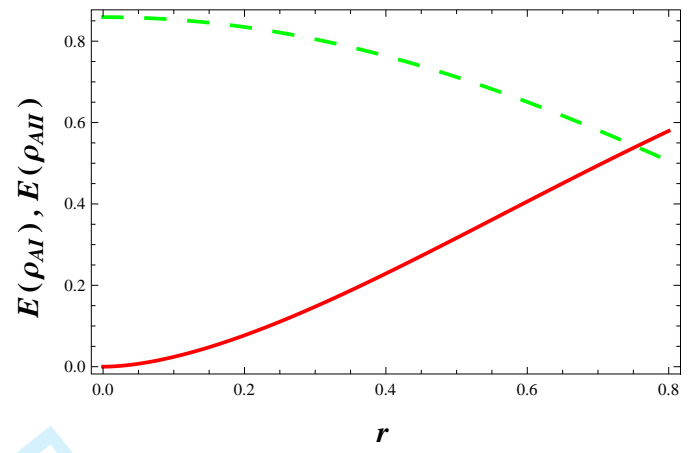

Figure 13: Comparison of EOF for $\rho_{A I}$ (green dashed) and $\rho_{A I I}$ (solid red) with $\alpha=0.8$.
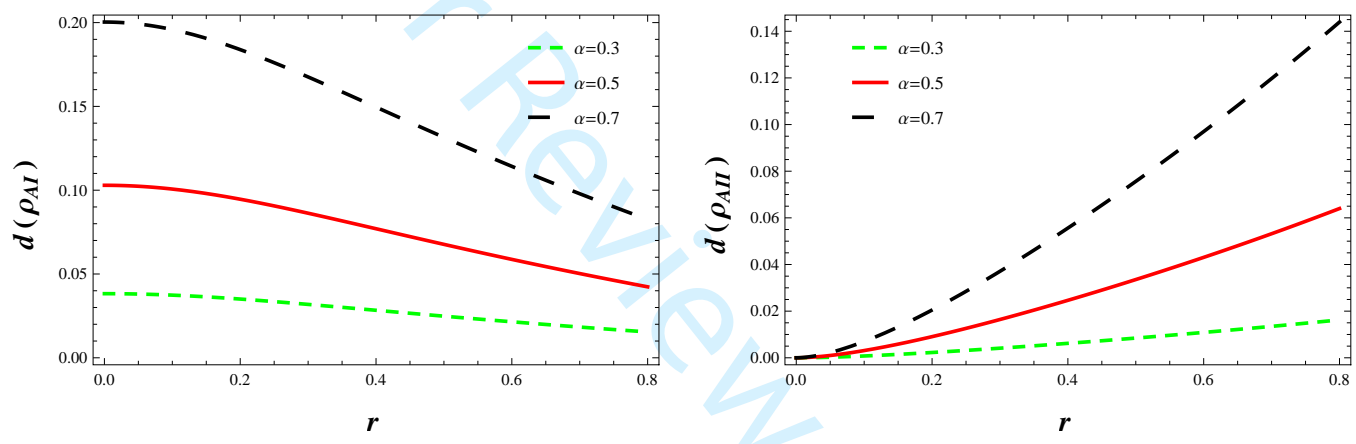

Figure 14: Quantum deficit of Alice-Rob (left) and Alice-antiRob (right) states versus $r$ for different values of $\alpha$ with $q_{R}=0.5$.
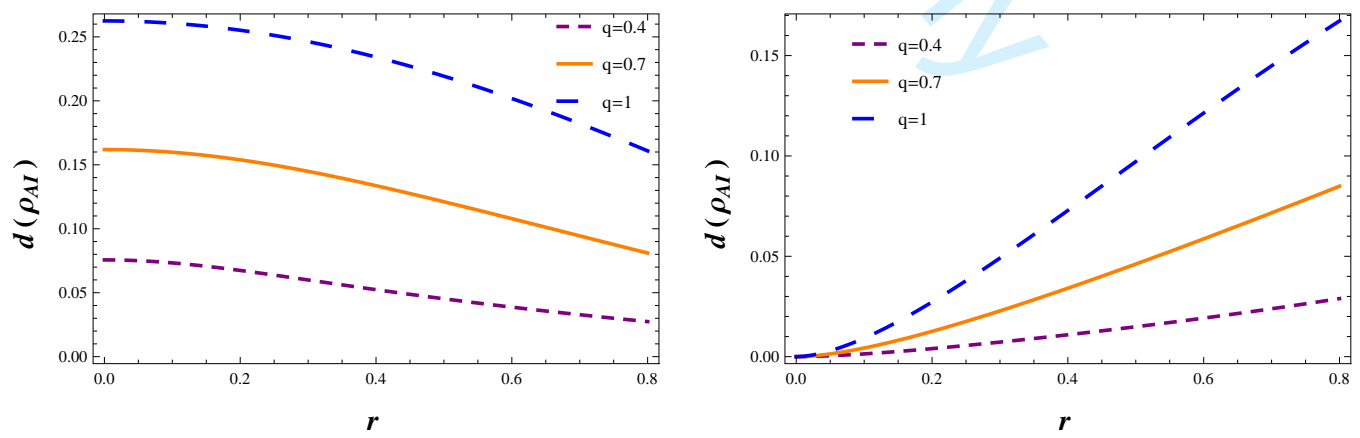

Figure 15: Quantum deficit of Alice-Rob (left) and Alice-antiRob (right) states versus $r$ for for different values of $q_{R}$ with $\alpha=0.5$. 

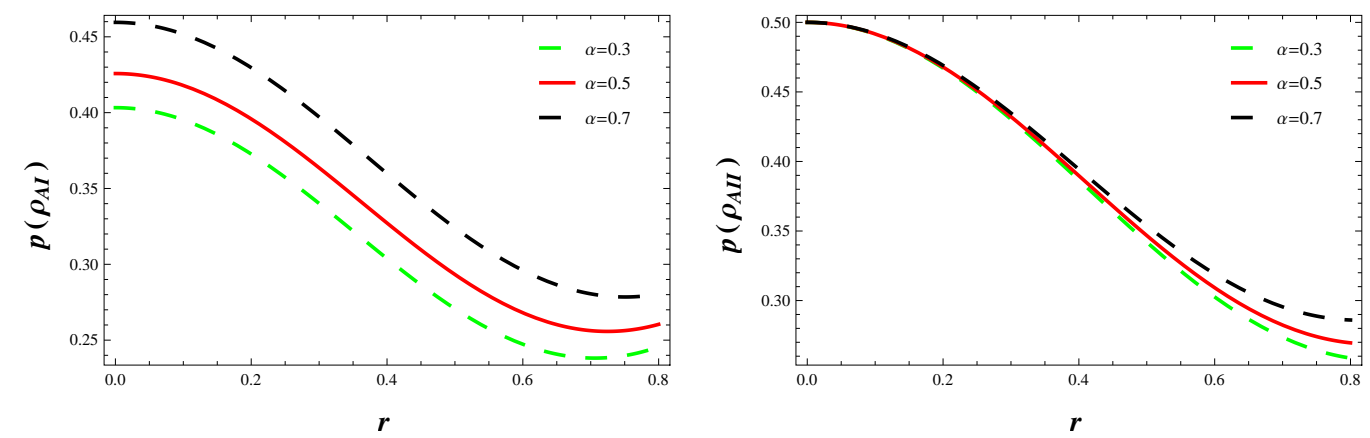

Figure 16: Purity of Alice-Rob (left) and Alice-antiRob (right) states versus $r$ for different values of $\alpha$ with $q_{R}=0.5$.
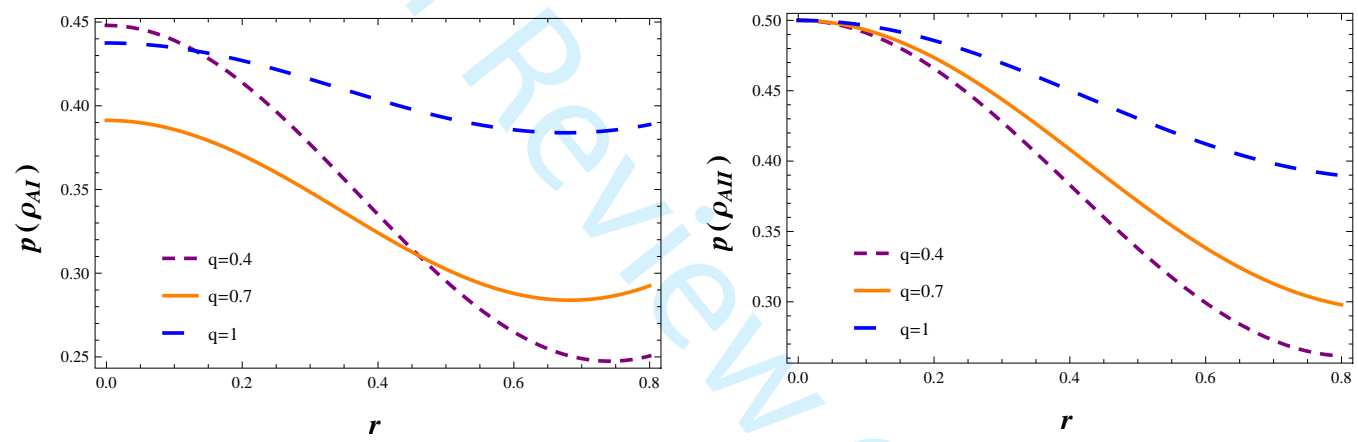

Figure 17: Purity of Alice-Rob (left) and Alice-antiRob (right) states versus $r$ for for different values of $q_{R}$ with $\alpha=0.5$.
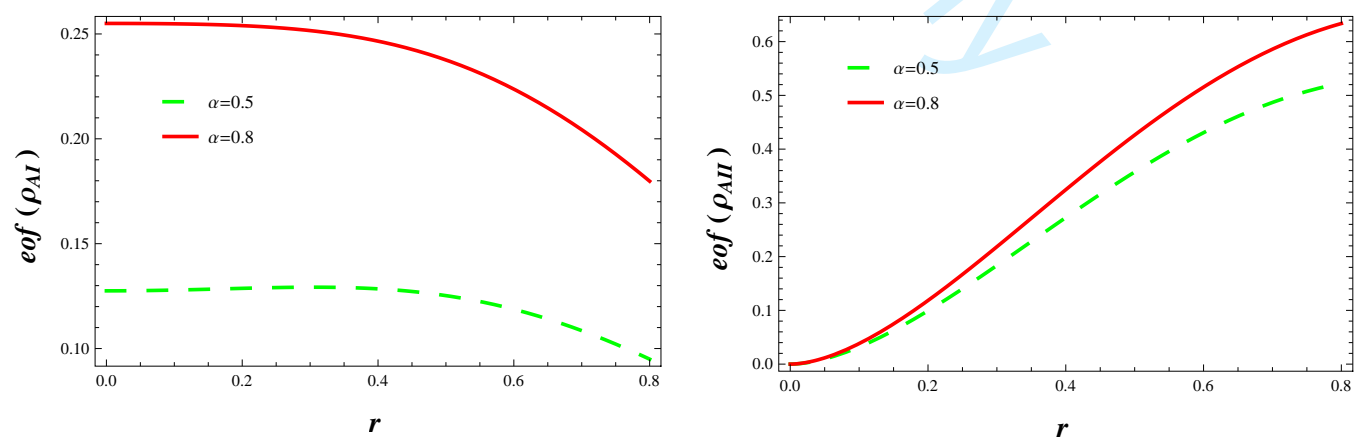

Figure 18: Entanglement of Alice-Rob (left) and Alice-antiRob (right) states versus $r$ for different values of $\alpha$ with $q_{R}=0.5$. 

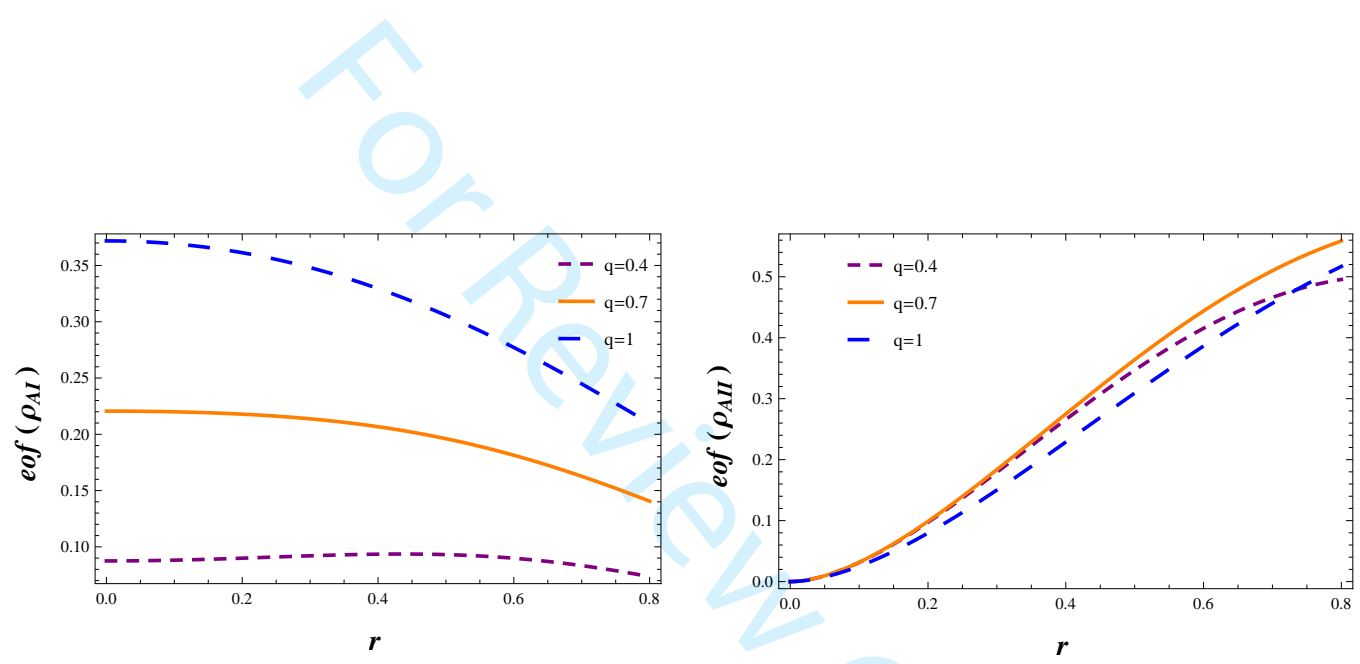

Figure 19: Entanglement of Alice-Rob (left) and Alice-antiRob (right) states versus $r$ for for different values of $q_{R}$ with $\alpha=0.5$. 